\title{
OLZ
}

\section{China und die Wissenschaft im frühen 20. Jahrhundert ${ }^{1}$}

von Angelika C. Messner

Trotz mehrfacher Zugeständnisse - wie etwa die Öffnung des chinesischen Kernlandes für Missionare nach 1860 oder die rechtliche Extraterritorialität der Ausländer - lässt sich die neuere Geschichte Chinas weder allein in Begriffen von Imperialismus und Kolonialismus fassen, noch lässt sich das Geschick Chinas zu Zeiten aggressiver Expansionsbestrebungen allein in Begriffen von Souveränität oder Unabhängigkeit beschreiben. Nichtsdestotrotz, obgleiches China gelungen ist, die Einflussmöglichkeiten der ausländischen Mächte auf ein Mindesmaß zu beschränken, lässt sich die neuere $\mathrm{Ge}$ schichte Chinas nicht ohne Bezugnahme auf die westlichen Mächte - spätestens seit den Opiumkriegen (1840-42) - beschreiben. Vor allem auch lässt sich die neuere Geschichte des Austauschs zwischen China und der westlichen Welt nicht ohne Bezugnahme auf die „Wissenschaft" begreifen, jenes Kulturprodukt, welches als Garant für die Befreiung Chinas aus dem Desaster, in dem es sich spätestens seit 1895 wähnte, gehandelt wurde. Nachdem China im Konflikt um die Oberhoheit in Korea eine empfindliche Niederlage gegen Japan hinnehmen musste und diese Niederlage als Verlust der chinesischen Souveränität in wirtschaftlicher, politischer sowie militärischer Hinsicht im Vertrag von Shimonoseki (1985) festgehalten wurde, setzten die ausländischen Mächte China verstärkt unter Druck, in dessen Folge in den Vertragshäfen Industriebetriebe aufgebaut sowie ausländisch finanzierte Eisenbahnnetze das Land zu erschließen begannen. Die Forderung nach einer eigenen Industrie, einer Armee nach westlichen Vorbildern, einem modernen Postwesen sowie einem staatlichen Bankensystem war Teil der von Kang Youwei 康有爲 (1858-1927) und Liang Qichao 梁棨超 (1873-1929) ausgearbeiteten Reformvorschläge, die 1898 zwar erlassen, doch nach kurzer Zeit wieder eingestellt (100-TageReform) wurden. Die im Anschluss an den Boxer-Aufstand $(1900)^{2}$ eingeforderten Entschädigungszahlungen

1 Zugleich Besprechung von: Martina Eglauer. Wissenschaft als Chance: Das Wissenschaftsverständnis des chinesischen Philosophen Hu Shi (1891-1962) unter dem Einfluss von John Dewey (1859-1952) Pragmatismus. [Münchener Ostasiatische Studien. Band 79] Stuttgart: Franz Steiner Verlag, 2001.

2 Dieser Aufstand ist als Folge vielfältiger Faktoren wie Flutund Dürrekatastrophen in Nord-China und im Anschluss an die Besetzung von Qingdao und dessen Hinterland durch deutsche Besatzungsmächte ausgebrochen. Insgesamt kamen in dessen Verlauf 229 an die acht Großmächte erreichten zwischen 1901 und 1910 etwa die Hälfte des Gesamt-Budgets Chinas. In Reaktion darauf sollten Reformen (1905) eine GrundErneuerung Chinas herbeiführen. ${ }^{3}$

Das vorliegende Buch beschreibt die genannten Vorfälle nicht explizit, wohl aber sind diese von unmittelbarer Relevanz für die verschriftlichten Diskurse, die hier untersucht werden. Eglauers Monographie gehört zu den Büchern, die bei näherem Hinsehen nicht leicht und ad boc in eine einzelne Sparte einzuordnen sind. Geschichte der Philosophie, Politikgeschichte, Wissenschaftsgeschichte, Geschichte der Kulturkontakte, des Kulturtransfers und der „Verhandlung“ von Identitäten, Geschichte der Übersetzung (Übersetzungsgeschichte): um nur einige wenige zu nennen. Es ließe sich aber auch einfach "chronologisch" in die "Republikzeit" (1912-1949) einordnen. Damit allerdings wären zwar nicht einmal die Lebensspannen der Protagonisten des Buches - Hu Shi 胡適 (1891-1962) und John Dewey (1859-1952) - abgedeckt, gleichwohl allerdings deren maßgebliche Zeit des persönlichen Wirkens.

Die Autorin aber lenkt den Leser unmerklich auf die Fährte, die sie gelegt hat und die sie mit Beharrlichkeit verfolgt: Es geht um die Bedeutung, die die „Wissenschaft" (kexue 科學) in den Köpfen einiger herausragender Denker und Aktionisten inmitten der mannigfaltigen (intellektuellen, politischen und sozialpolitischen) Wirren des frühen 20. Jahrhunderts auf dem chinesischen Festland eingenommen hat. Damit nimmt sie eine vergleichsweise strenge Engführung vor. ${ }^{4}$ Dass die Begriffe "Wissenschaft", kexue bzw. science, jeweils ihre Geschichte haben, und die Übersetzung spezifischer Ansätze und Methodologien von einer Welt in eine andere und von einer Sprache in eine andere eine höchst komplexe Angelegenheit war und ist, die unzählige

Ausländer zu Tode. Vgl. insbesondere Joseph W. Esherik. The Origins of the Boxer Uprising. Berkeley, Los Angeles, London: University of California Press, 1987.

3 Die Abschaffung der Beamtenprüfungen etwa bewirkte, dass ein Studium im Ausland attraktiv wurde. Der Aufruf zur Erneuerung des Erziehungssystems lief letztlich auf eine Erneuerung des chinesischen Menschen hinaus. Siehe hierzu Douglas R. Reynolds. China, 1898-1912. The Xinzheng Revolution and Japan. Cambridge, Mass., Harvard University Press, 1993.

${ }_{4}$ Im Vergleich zu anderen, ähnlich gelagerten Monographien über den entsprechenden Zeitraum. In diesem Zusammenhang sei allein auf eine kürzlich im deutschen Sprachraum erschienene, bemerkenswert pointiert und lesbar geschriebene, Arbeit hingewiesen: Thomas Fröhlich. Staatsdenken im Cbina der Republikzeit (1912-1949): Die Instrumentalisierung philosophischer Ideen bei chinesischen Intellektuellen. Frankfurt, New York, Campus Verlag, 2000. 
Zweige und Verästelungen von „Vorwissen“ mit einbeziehen kann oder muss - davon handelt dieses Buch, und es exemplifiziert anhand des untersuchten Materials die solchen Übersetzungsprozessen inhärenten gewaltigen Unwegsamkeiten und Widersprüchlichkeiten.

Hu Shi 胡適 (1891-1962) hat zwischen 1910 und 1917 in Amerika studiert ${ }^{5}$ und bereits während dieser Zeit im Ausland großen Einfluss auf die Neue Kulturbewegung (Xin wenhua yundong 新文化運) ausgeübt, die sich um 1915 aus den zahllosen Diskussionen und Debatten um die Errettung des Vaterlandes herausschälte. Die Autorin beschränkt sich zunächst auf seine Schriften aus den Jahren 1917-1922. 1917 war das Jahr der Fertigstellung seiner Dissertation und das Jahr, in dem er, nach sieben Jahren Studium in Nordamerika, nach China zurückkehrt und einen Ruf als Philosophieprofessor (für die folgenden neun Jahre) an der Peking-Universität annimmt. 1919 kam John Dewey (1859-1952) für zwei Jahre nach China, um an diversen Universitäten Vorlesungen zu halten. Die Jahre zwischen 1917 und 1922 widmet $\mathrm{Hu}$ Shi vorwiegend der Übertragung der Ideen des Pragmatismus und der wissenschaftlichen Methode ins Chinesische. $\mathrm{Ab}$ den 1930er Jahren befasst er sich verstärkt mit der chinesischen Philosophie und deren Aufarbeitung für westliche Rezipienten.

Diskutiert wurde nun nicht mehr nur das Verhältnis von (einseitigen) „Gebrauch (yong 用“) westlicher Tech-

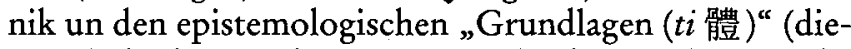
ser Technik), sondern nun wurde die Forderung, die „westlichen" Grundlagen zu übernehmen, programmatisch. In China war die erste Hälfte des 20. Jahrhunderts geprägt durch die Auseinandersetzung mit westlichen Konzepten von Leben und Zivilisation bzw. Fortschritt und Moderne; zugleich war sie geprägt durch Versuche, westliche Konzepte entweder fernzuhalten oder sie zu integrieren bzw. zu übernehmen.

Eglauer analysiert nicht nur in akribischer Weise die Geschichte der Übersetzung der verschiedenen termini technici, so u. a. die Genealogie etwa der Begriffe Lebensanschauung, Pragmatismus, oder Evolution - also ein Minenfeld von Begrifflichkeiten, die im gegenwärtigen chinesischen Kontext teilweise keine Rolle mehr spielen, nichtsdestotrotz aber während der ersten Dekaden des 20. Jahrhunderts im Spannungsfeld zwischen kultureller Eigenständigkeit und angestrebter Modernisierung von signifikanter Bedeutung gewesen waren. Darüber hinaus beleuchtet sie auch Hintergründe: Wie dringlich die Auseinandersetzung mit westlichen Konzeptionen von Demokratie und Liberalismus für chinesische Intellektuelle des frühen 20. Jahrhundert war, davon zeugen nämlich die heiß geführten Kontroversen "Über östliche und westliche Kultur" sowie über "Wissenschaft und Lebensanschauung" (1922/23, ausgelöst von einer Rede des Zhang Junmai 張君勱 (1886-1969)

5 Er ging mit dem so genannten Boxer-Stipendium zunächst an die Cornell-University in Ithaca, um Agrarwissenschaften zu studieren, um sich dann, ein Jahr später, für Philosophie zu entscheiden und um, wiederum ein Jahr später, Pädagogik bei John Dewey zu studieren. Siehe Eglauer 2001, S. 24. an der Qinghua-Universität 1922), wobei Fragen der Verwestlichung der chinesischen Kultur und der Verwissenschaftlichung des chinesischen Denkens diskutiert wurden. Die Umsetzung der alles Traditionelle negierenden Sicht stellte sich in den jeweiligen Lebensrealitäten der Protagonisten viel komplexer dar, als diese es möglicherweise wahrhaben wollten. Dass die in und außerhalb Chinas vonseiten chinesischer Intellektueller geführten Diskussionen und Debatten um die Errettung des Vaterlandes beginnend mit dem späten 19. Jahrhundert bis in die späten 1940er Jahre hinein wesentlich um Wissenschaft kreisten, gehört mit Sicherheit zum Allgemeingut eines jeden mit der Geschichte Chinas Befassten. Hierzu gehört auch das Wissen darum, dass der Pragmatismus kaum Anklang unter chinesischen Intellektuellen gefunden hat. Im Falle von $\mathrm{Hu}$ Shi war die Sache höchst komplex, wie Eglauer zeigt, denn zum einen vermeidet $\mathrm{Hu}$ Shi eine aktivistische Haltung im Bemühen um eine Erneuerung der chinesischen Gesellschaft. Zum anderen aber fordert er dezidiert, und damit nicht minder bilderstürmerisch als so manch revolutionärer Geist zu jener Zeit, die Übernahme derjenigen Maßstäbe, die er während seines Studiums in Nordamerika kennengelernt hatte und die er als "Wundermittel“ für den Neuaufbau Chinas ansieht. (Teil III, S. 105-148).

Nach 1917, als er als Philosophieprofessor an der Beijing Universität lehrte, setzte $\mathrm{Hu}$ Shi sich für die Literaturreformbewegung ein, wobei er sich insbesondere für die Umgangssprache (baibua 白話) als Medium in der Literatur stark machte. $\mathrm{Hu}$ Shi gehörte damit zu den prominentesten Figuren im Vorfeld der Vierten MaiBewegung (Wusi yundong 五四運動), u. a. neben $\mathrm{Li}$ Dazhao 李大釗 (1889-1927), Kang Youwai 康有爲 (1858-1927), Liang Qicho 梁棨超 (1873-1929), Ding Wenjiang 丁文江 (1887-1936), Zhang Junmai 張君勱 (1886-1969) sowie Chen Duxiu 陳獨秀 (1879-1942). Welche Rolle spielte diese Bewegung für die Neue Kultur im Hinblick auf die Umbrüche und Wandlungen im frühen 20. Jahrhundert und welche Rolle spielten wiederum die Debatten "für oder wider" die Wissenschaftliche „Lebensanschauung“ für diese Bewegung, die in den 1920er Jahren zur Bühne und „Integrationsfigur“ vieler unterschiedlicher "-Ismen " und Ideen geworden war? Nationalismus, Liberalismus, Anarchismus, Individualismus, Darwinismus, Sozialismus, Marxismus und Pragmatismus. Der Transfer all dieser Ismen wurde begleitet von und unterfüttert mit dem Kampf gegen die Tadition, insbesondere gegen den Konfuzianismus, wobei $\mathrm{Hu}$ Shis „Nieder mit dem Konfuzius-Laden (Dadao Kongjiadian 打倒孔家店)“ zum geflügelten Wort avancierte.

Es versteht sich von selbst, dass eine Dissertation solche Gemeinplätze verlassen muss, wenn sie die darunter liegenden Spuren von Widersprüchlichkeiten und Ambivalenzen nicht ausblenden will, ohne die die Auseinandersetzung mit neuen Denk- und Handlungsweisen nicht möglich gewesen zu sein schien. Die Autorin verfolgt die vielen Wege, Seitenwege und selbst kleinste Verästelungen derjenigen Denkansätze, die bei $\mathrm{Hu}$ Shi eine Rolle spielten. 
John Dewey, bei dem $\mathrm{Hu}$ Shi mehrere Jahre studiert hat, gilt als Begründer und Vertreter des Instrumentalismus, einer spezifischen Ausprägung des auf Charles S. Peirce, William James und Chauncy Wright zurückgehenden Pragmatismus. Der Genese dieser Denkrichtung als wissenschaftliche Methode in der Philosophie widmet die Autorin annähernd 50 Seiten (Teil II, S. 57-104). Sie will Deweys Entwicklung als Philosoph und als Pädagoge nachzeichnen, und dies macht sich bezahlt, denn so manche Widersprüchlichkeit und so mancher heute als anachronistisch erscheinender Gedanke bei $\mathrm{Hu}$ Shi - ist in John Deweys Denken zu finden. So begreift er "Wissenschaft" als rein formal und moralisch neutral, setzt die "experimentelle Methode" mit der "wissenschaftlichen" Methode gleich und spricht dabei von einer wissenschaftlichen Haltung, die allein in demokratisch ausgerichteten Gesellschaften eine mögliche Entfaltung erfahren könnten. Dabei begreift er die Demokratie als "persönliche Lebenshaltung", die den permanenten Einsatz für die demokratische Idee erfordert.

Eglauer präsentiert weitläufige Übersetzungsausschnitte aus dem Chinesischen und alteriert diese durch - von $\mathrm{Hu}$ Shi verfassten - englische Textausschnitte. Dadurch wird die Mehrschichtigkeit der Verhandlungsebenen bei Hu Shi explizit gemacht. Auf welche Begrifflichkeiten griff $\mathrm{Hu}$ Shi zurück, wenn er die im Dewey'schen Pragmatismus signifikanten Begrifflichkeiten ins Chinesische übertrug? Welche termini technici entwickelte er, um sich möglichst nahe an den „ursprünglichen“ Bedeutungen zu bewegen? In welchen Zusammenhängen wählte und benutzte er möglichst "simple“ Explikationen, sowohl hinsichtlich der westlichen Zivilisationen als auch hinsichtlich der chinesischen? Was sagen solch dezidierte Vereinfachungen über $\mathrm{Hu}$ Shis Unternehmen aus? Enthüllen sie etwas von den mannigfaltigen Schwierigkeiten, während der 1930er Jahre als chinesischer Intellektueller in den Vereinigten Staaten Anerkennung und Wertschätzung zu erlangen und zwar vor dem Hintergrund der gewaltigen Krisen im eigenen Lande? Enthüllen sie auch etwas von den Unwegsamkeiten, denen $\mathrm{Hu}$ Shi begegnete, weil er die chinesische Kultur auf eine höhere Ebene heben wollte und dabei die Außenperspektive zu sehr ins Blickfeld nahm bzw. diejenige John Deweys? Die spezifische Art und Weise, wie Hu Shi den Dewey'schen Pragmatismus zur "universell“ gültigen Methode erhebt, womit indigene Traditionalismen aufgehoben, negiert, ad acta gelegt und durch neue, aktive, funktionelle Grundsätze ersetzt werden konnten - dieses spezifische Ansinnen basierte wesentlich auf dem Grundsatz der „Objektivität“ von Wissenschaft. Wissenschaft als unantastbare, unangreifbare Größe, die bar jeglicher Glaubenssätze und bar jeglicher Eigeninteressen besteht und zum Gedeih jeder menschlichen Gesellschaft wird, wenn man sie nur richtig versteht und einsetzt. Das richtige Verständnis von Wissenschaft sei somit auch die Voraussetzung für die Reorganisation der chinesischen Geschichte und der darauf aufbauenden Reorganisation der chinesischen Zivilisation (Teil V, S. 216-264).

$\mathrm{Hu}$ Shi bewegte sich immer wieder in Zwischenräumen, die sich auf dem Parkett des Transfers einer ganz bestimmten Ausprägung des Pragmatismus bzw. des Instrumentalismus in den chinesischen Kontext auftaten. Dieser Transfer bedeutete selbstredend nicht allein die Übersetzung von einzelnen Wörtern in die andere Sprache. Obgleich Hu Shi John Dewey als seinen Lehrer und als Begründer dieser Denkrichtungen (Methoden, Wissenschaftsverständnis) hoch schätzte und eigentlich "nur" dessen Ideen für China fruchtbar machen wollte, konnte es nicht ausbleiben, dass dieser Prozess des Transferierens eigene Schöpfungen hervorbrachte. Die Autorin beschreibt diese Prozesse des Hevorbringens „neuer“ Formationen der „wissenschaftlichen Methoden in der Philosophie" unter $\mathrm{Hu}$ Shis Federführung, indem sie etwa seinen diversen Ausflügen in die chinesische Frühzeit folgt, in der er chinesische (indigene) Frühformen des Evolutionsgedankens nachzuweisen sucht. (Teil IV, S. 180-212). Sie lässt sich auf die mannigfaltigen Vagheiten und Volatilitäten ebenso ein wie auf die aus der heutigen Perspektive betrachtet - Anachronismen. Damit ermöglicht sie die Sichtung mancher - bislang im Dunkeln verbliebener - Facetten dieser höchst aufregenden Übersetzungsgeschichte. Dazu gehört der Befund, dass $\mathrm{Hu}$ Shi nicht nur "pragmatistisches" Denken in die chinesische Diskussionslandschaft einführt, in dessen Folge heftige Diskussionen (wie etwa die Debatte "Wissenschaft und Lebensanschauung") um die Modernisierung Chinas geführt wurden, sondern dass er außerdem und gleichermaßen die westliche, insbesondere nordamerikanische intellektuelle Rezeption chinesischer Geschichte und Philosophie kannte. (Teil VI. $\mathrm{Hu}$ Shis Position innerhalb zeitgenössischer Debatten, S. 265-311) Hu Shi erfüllte somit eine Pivotal-Funktion für beide kulturellen Kontexte, in denen er sich bewegt hatte. Seine Überzeugung, dass die Erneuerung Chinas nur auf unpolitischem Wege bzw. nur auf kulturellem Wege erreicht werden könne, war die Basis für sein immenses Engagement und natürlich auch für die Kampagnen, die zu Beginn der 1950er Jahre vonseiten der Kommunistischen Partei Chinas gegen ihn geführt wurden, weil man ihm vorwarf, als Lakai von Jiang Jieshi 將介石 (Chiang Kai-shek, 1887-1975) gedient zu haben.

Angesichts der Dringlichkeit, die die Beschäftigung mit Wissenschaft in Hu Shis umfassendem Schrifttum offensichtlich einnimmt, hat die Autorin es sich zur Aufgabe gemacht, das Verständnis von Wissenschaft bei Hu Shi unter die Lupe zu nehmen. Dieser spezifische Fokus fehlt in den bis dato vorgelegten Arbeiten $z \mathbf{z u} \mathrm{Hh}$. Das vorliegende Buch vermag diese Lücke zu schließen. Der Zusammenhang von Wissenschaft, wissenschaftlicher Methode, Pragmatismus, Instrumentalismus und Evolutionismus sowie der Erneuerung der Kultur besteht bei $\mathrm{Hu}$ Shi in der Betonung einer wissenschaftlichen Haltung, womit er eine gegenteilige Haltung zu konservativen und fatalistischen Lebensentwürfen meint.

Der verschriftlichte Diskurs $\mathrm{Hu}$ Shis zieht sich wie ein roter Faden durch das ganze Buch. Dadurch wird die höchst komplexe und vielschichtige Verfasstheit der politischen, sozialen und geistigen Bewegung und Umbrüche im China des frühen 20. Jahrhunderts nicht unbedingt 
vereinfacht, aber, so sich der Leser auf das unermüdliche Beharren der Vision Hu Shis einer (universell) verfassten Kultur und Zivilisation einlässt, eine klare Linie sichtbar. Die Tatsache, dass sich die - von $\mathrm{Hu}$ Shi erarbeiteten Begrifflichkeiten keineswegs als konsistent verfasstes Design erweisen, sondern höchst widersprüchliche Züge aufweisen, wurde häufig und in unterschiedlichen Zusammenhängen moniert. Allem Anschein nach bedurfte es einer spezifischeren Perspektive, und zwar von der Art, die Martina Eglauer in ihrem Buch eingenommen hat, um diese Widersprüchlichkeiten letztendlich als Sinnhaftigkeit entpuppen zu können. Denn, die Rede von einer Weltkultur, in der westliches und östliches Denken zu einer Weltphilosophie verschmelzen werden, $d . h$. in der kulturelle Differenzen durchaus „harmonieren“ können, bekommt in der Umschreibung „pluralistische Essenz des Pragmatismus "6 eine höchst aktuelle und alles andere als widersprüchliche Pointierung.

\section{Ägyptologie}

Spencer, A. J.: Excavations at Tell el-Balamun 1999-2001. London: British Musem Press 2003. 56 S., 4 Farbtafeln, 47 Schwarzweißtafeln. $4^{\circ}$ Brosch. $£ 60.00$. ISBN 0-7141-1958-X. - Bespr. von Robert Schiestl, Berlin.

A. J. Spencer legt mit diesem Band den dritten Grabungsbericht aus Tell el-Balamun, dem antiken SmaBehet-Painenamun, der nördlichsten Stadt Ägyptens, vor. Der Autor leitet die Ausgrabungen des British Museums London seit 1991. Dieser Band berichtet auf 46 Seiten, mit 51 Tafeln, über die Arbeit der Jahre 1999-2003 in und um den Tempel des Gottes Amun (Tempel A). Kapitel 1 behandelt die Untersuchung der ramessidischen Umfassungsmauer, Kapitel 2 einen Elitefriedhof der 22. Dynastie, der nördlich des Tempels errichtet wurde, und Kapitel 3 umfasst Kurzberichte über Untersuchungen an einem Prozessionsweg, sowie Arbeiten im Bereich des nordöstlich gelegenen Tempels $B$. Es folgt in Kapitel 4 ein Katalog der Stein-, Metall-, Fayence- und Keramikfunde. Joyce Filer verfasste den Bericht über die menschlichen und tierischen Skelettreste in Kapitel 5. Das Buch schließt mit einer kurzen Zusammenfassung der Ergebnisse in Kapitel 6. Als Appendix folgt noch das Inventar einer ptolemäischen Grube, welche in eines der Gräber einschneidet.

Die Untersuchung der ramessidischen Umfassungsmauer in Kapitel 1 schließt unmittelbar an die Ergebnisse der letzten Publikation an (Vgl. A. J. Spencer, Excavations at Tell el-Balmun 1995-1998, London 1999, plate 2). Die Mauer ließ sich im Nordosten, vor der Tem-

6 Vgl. Martina Eglauer, ",Pluralistisches Universum - Die pluralistische Essenz des Pragmatismus von Hu Shi (1891-1962)." In Eglauer, Martina Maria und Clemes Treter (Hrsg.), Einheit und Vielfalt in China. Beiträge zum Pluralismus in der chinesischen Geistesund Sozialgeschichte. [Jahrbuch der Deutschen Vereinigung für Chinastudien 1]. Wiesbaden, Harrassowitz Verlag, 2005. S. 107-125. pelfront, zur Gänze nachweisen. Der dort eingebaute Pylon war aus Kalkstein errichtet gewesen und nur mehr durch eine dunkle Schlammverfüllung des Fundamentgrabens nachweisbar, da sämtliche Kalksteinbauteile des Tempels geraubt worden waren. Westlich und östlich der Tempelachse konnten noch Abschnitte der nach Süden verlaufenden Umfassungsmauer erfasst werden. Während die Umfassungsmauer im Westen in relativ knappem Abstand zum Tempelgebäude verläuft (zur Achse des Tempels beträgt der Abstand zwischen 52 und $53 \mathrm{~m}$, also 100 Ellen), war der Abstand im Osten deutlich größer. Um die Annahme, dass dieser Platz der Errichtung von Nebenbauten gedient hatte, zu verifizieren, wurden zwei Testschnitte angelegt. Dabei wurden jedoch keine Spuren von Bauten der Ramessidenzeit entdeckt, sondern nur eine ptolemäische Abfallgrube. Im Bereich der beiden untersuchten Ecken der ramessidischen Umfassungsmauer lässt sich die weitere Geschichte des Areals gut verfolgen. Der Verfall der Mauern setzte rasch ein und eine domestische Nutzung des Areals ist schon für die 21. Dynastie nachweisbar. Das Nordosteck wurde von Häusern überbaut (plate 11), deren vorwiegend keramisches Inventar ausführlich abgebildet wird (plates 15-17). Noch in der 3. Zwischenzeit werden diese Bauten durch eine neue Umfassungsmauer überbaut. Diese Mauer, die nur in ihrem Nordosteck dokumentiert ist, erweitert den Abstand zum Tempelgebäude um ein weiteres Stück. Es lässt sich für diesen Bereich beobachten, was schon für andere Teile des Tempelareals feststellbar war: Der sakrale Bereich wird stetig expandiert, auf Kosten von Wohngebieten, die immer näher an das Tempelgebäude gebaut worden waren. Der Höhepunkt dieser Entwicklung ist mit der Umfassungsmauer der 30. Dynastie erreicht. Auch das Nordwesteck der ramessidischen Umfassungsmauer weist einen raschen Verfall und Spuren einer domestischen Nutzung auf. In der 22. Dynastie wird jedoch dieser Bereich für die Anlage eines Elitefriedhofes genutzt, wovon Kapitel 2 handelt. Durch die hier vorgelegten neu entdeckten Grabkomplexe 2 und 3 wurde erkennbar, dass das 1998 gefundene und im darauf folgenden Jahr publizierte Grab des Iken (Spencer 1999) Teil eines kleinen Grabbezirks ist. Die Lage der Gräber am Tempel ist ein ab der 3. Zwischenzeit archäologisch belegter Brauch, deren bekannteste Vertreter die Königsgräber von Tanis bilden. Wenn auch Beispiele aus Oberägypten vorliegen (Medinet $\mathrm{Habu}$ ), so ist dennoch eine Entstehung dieses Grabbrauchs aus einer älteren Deltatratition vorstellbar. Jüngst wurde von Thomas Schneider ${ }^{1}$ vorgeschlagen, dass die Bestattungen zweier Könige des ausgehenden Neuen Reiches, Ramses VIII. und X., in einem Tempelbezirk von Pi-ramesse, dem im Delta gelegenen Sitz der Ramessiden, zu suchen wären. Eine zusammenfassende Darstellung zum Grabbrauchtum dieser Epoche liegt mit der Arbeit von José Lull vor. ${ }^{2}$

1 Thomas Schneider, in: Hanna Jenni (Hg.) Das Grab Ramses' X. (KV 18), Aegyptiaca Helvetica 16, Basel 2000, 104-108.

2 José Lull, Las tumbas reales egipcias del Tercer Período Intermedio (dinastías XXI-XXV). Tradición y cambios, BAR International Series 1045, Oxford 2002. 